\title{
CONSTRUÇÃO E IMPLEMENTAÇÃO DE AÇÕES DE ENFERMAGEM EM AMBULATÓRIO DE GASTROENTEROLOGIA
}

\author{
Cristina Hitomi Kubo' \\ Patrícia de Jesus Ribeiro ${ }^{1}$ \\ Luciane Aparecida Köpke de Aguiar? \\ Carlos Fischer de Toledo ${ }^{3}$ \\ Sônia Maria de Oliveira Barros ${ }^{4}$ \\ Durval Rosa Borges ${ }^{5}$
}

Kubo CH, Ribeiro PJ, Aguiar LAK, Toledo CF, Barros SMO, Borges DR. Construção e implementação de ações de enfermagem em ambulatório de gastroenterologia. Rev Latino-am Enfermagem 2003 novembro-dezembro; 11(6):816-22.

Em 1998, iniciou-se reestruturação do Setor de Esquistossomose da Disciplina de Gastroenterologia da UNIFESP, com a implantação do Serviço de Enfermagem. Objetivo: desenvolver ações administrativas de enfermagem, sistematização da assistência e programa de orientação ao esquistossomótico. Método: estudo descritivo, realizado no Ambulatório de Gastroenterologia que atende portadores de esquistossomose, nas suas diferentes formas clínicas. Resultados e discussão: a reestruturação das atividades do Setor resultaram em: criação de estrutura organizacional e implantação da consulta de enfermagem; criação e distribuição dos folhetos explicativos e desenvolvimento do Programa de Educação em Esquistossomose; início de projeto piloto de banco de dados de todos os clientes do ambulatório e do cadastro eletrônico da coleta de materiais biológicos. Conclusão: a iniciativa abriu nova oportunidade de atuação para o enfermeiro e contribuiu para melhorar a qualidade do atendimento ambulatorial.

DESCRITORES: esquistossomose mansoni; assistência ambulatorial; diagnóstico de enfermagem; educação em saúde

\section{CONSTRUCTION AND IMPLEMENTATION OF NURSING ACTIONS IN THE} GASTROENTEROLOGY OUTCLINIC

The reorganization of the Schistosomiasis Sector of Gastroenterology at UNIFESP started in 1998, when the Nursing Service was implanted. Objective: to develop administrative nursing actions, systematization of care and orientation program for the schistosomic patient. Method: a descriptive study carried out at the Gastroenterology Outclinic, which attends schistosomic patients of different clinical types. Results and Discussion: the reorganization of activities in this Sector resulted in the creation of an organizational structure and the implantation of the nursing visit; creation and distribution of explanatory leaflets and development of the Schistosomiasis Education Program; beginning of the database pilot project with all outpatients and an electronic register of the collection of biologic material. Conclusion: this initiative opens up a new opportunity for nursing actions and contributes to quality improvement in ambulatory care.

DESCRIPTORS: schistosomiasis mansoni; ambulatory care; nursing diagnosis; health education

\section{CONSTRUYENDO Y IMPLEMENTANDO ACCIONES DE ENFERMERİA EN LA CONSULTA EXTERNA DE GASTROENTEROLOGÍA}

En 1998, empezó la reestructuración del Sector de Esquistosomiasis de la Disciplina de Gastroenterología de la UNIFESP, con la implantación del servicio de enfermería. Objetivo: desarrollar acciones administrativas de enfermería, sistematización de asistencia y programa de orientación esquistosomótico. Método: estudio descriptivo, realizado en la consulta externa de Gastroenterología que atiende portadores de esquistosomiasis en las distintas formas clínicas. Resultados y Discusión: las reestructuración de las actividades del Sector resultaron en: la creación de la estructura organizacional y la implantación de la consulta de enfermería; la creación y distribución de panfletos explicativos y el desarrollo del Programa de Educación en Esquistosomiasis; inicio del Proyecto Piloto del banco de datos de todos los pacientes de la consulta externa y del registro electrónico de la recolección de materiales biológicos. Conclusión: la iniciativa trajo una nueva oportunidad de actuación para el enfermero y contribuyó para mejorar la calidad en la atención de la consulta externa.

DESCRIPTORES: esquistosomiasis mansoni; atención ambulatoria; diagnóstico de enfermería; educación en salud

\footnotetext{
${ }^{1}$ Enfermeira, Pós-Graduanda da Universidade Federal de São Paulo, e-mail: cris_kubo@hotmail.com; ${ }^{2}$ Médica, Mestre; ${ }^{3}$ Professor Adjunto;

${ }^{4}$ Professor Adjunto, Livre-Docente; ${ }^{5}$ Professor Titular. Universidade Federal de São Paulo
} 
INTRODUÇÃO

Este estudo tem o propósito de apresentar a reestruturação do Setor de Esquistossomose da Disciplina de Gastroenterologia da Universidade Federal de São Paulo (UNIFESP), iniciada em agosto de 1998. Destaca o significado e a importância de a administração estar relacionada às formas de tornar o trabalho de equipe de saúde mais fácil e mais bem sucedido.

A administração dos serviços de saúde é processo dinâmico e deve ser sempre repensada, para ganhar eficácia, em sua sistemática, estrutura, processos e resultados. Desse modo, ela não é feita somente para o benefício da instituição, mas também para o melhor atendimento dos que utilizam seus serviços. $\mathrm{Na}$ enfermagem, como em outras profissões, o profissional incorpora, em sua formação, o saber de várias disciplinas, por exemplo, a disciplina de administração ${ }^{(1)}$.

O administrador é o responsável pelo desempenho de uma organização. Ele planeja, organiza, dirige pessoas, gera e controla recursos materiais, financeiros, de informação e tecnologia, visando à realização de determinados objetivos ${ }^{(2)}$.

Uma das primeiras funções administrativas é o planejamento, com vistas a estabelecer quais os objetivos e os meios de atingi-los. O planejamento da assistência de enfermagem é um dos meios de que o enfermeiro dispõe para aplicar os seus conhecimentos técnico-científicos e humanos na assistência ao cliente, e caracterizar sua prática profissional, colaborando na definição de seu papel $^{(3)}$.

A Disciplina de Gastroenterologia, da Universidade Federal de São Paulo, criada em 1947, atua em diversos setores especializados, laboratórios e linhas de pesquisas. Entre os diferentes setores da disciplina, o de Esquistossomose possui atividade de pesquisa, mas sua atividade assistencial era centrada na consulta médica. Como essa atividade assistencial não incluía programa de orientação aos pacientes portadores de esquistossomose que visasse atender suas necessidades, em 1998 iniciou-se a reestruturação do Setor, com a inclusão do enfermeiro na equipe de saúde.

$\mathrm{Na}$ rede básica de saúde, o enfermeiro tem a função de gerenciar a assistência de enfermagem em ações educativas, preventivas e de vigilâncias epidemiológica e sanitária ${ }^{(4)}$. Desse modo, o enfermeiro do Setor de Esquistossomose tornou-se responsável por organizar e estruturar o fluxo de atendimento do cliente, promovendo a divisão do trabalho da equipe de saúde. A implementação da assistência de enfermagem aos clientes portadores de esquistossomose teve como finalidade melhorar a qualidade e a resolutividade do atendimento.

Ao enfermeiro são atribuídas responsabilidade, autoridade e autonomia para desenvolver diferentes atividades entre elas, coordenação e gerenciamento do ambulatório, a consulta de enfermagem e atividades educativas. Desse modo, o enfermeiro colabora com a equipe multiprofissional (médicos, psicólogos, nutricionistas, entre outros) atuando como agente facilitador da comunicação e promovendo a continuidade do programa de prestação de cuidados ao cliente ${ }^{(5)}$.

O Art. ${ }^{\circ}$ - do decreto 94406 , de 1987 , faculta, como atividade privativa do enfermeiro, a consulta e a prescrição de enfermagem e, como integrante da equipe de saúde, a participação na elaboração, execução e avaliação dos planos assistenciais de saúde; a prescrição de medicamentos previamente estabelecidos em programas de saúde pública e em rotina aprovada pela instituição de saúde; e a participação em programas e atividades de educação sanitária, visando à melhoria de saúde do indivíduo, da família e da população em geral ${ }^{(6)}$.

De acordo com a decisão do Conselho Federal de Enfermagem (COFEN) ㄲo 001/2000, de 4 de janeiro de 2000, foi normatizada a implementação da Sistematização de Assistência de Enfermagem (SAE) nas instituições de saúde e no âmbito do Estado de São Paulo. Esse sistema já vem sendo utilizado em alguns serviços do Hospital São Paulo, do Hospital Santa Marcelina e do Ambulatório de Puericultura do Centro Assistencial Cruz de Malta ${ }^{(6)}$.

\section{OBJETIVOS}

- Divulgar as ações administrativas de enfermagem, visando à organização do atendimento e funcionamento do Setor de Esquistossomose.

- Implantar a Sistematização da Assistência de Enfermagem nesse setor.

- Desenvolver Programa de Educação em Esquistossomose aos clientes, à família e à comunidade.

\section{CASUÍSTICA E MÉTODO}

Tipo de estudo

Estudo descritivo sobre as ações de enfermagem 
implantadas em um ambulatório de Gastroenterologia, desenvolvidas nas áreas de gerenciamento, assistência, educação e pesquisa.

Local

Setor de Esquistossomose da Disciplina de Gastroenterologia da Universidade Federal de São PauloUNIFESP

População

Profissionais médicos e enfermeiros, psicólogo, alunos de graduação em Medicina e em Enfermagem e portadores de esquistossomose nas suas diferentes formas anatomoclínicas. Esse setor tem cadastrados cerca de 1000 clientes.

Ações de enfermagem

Apresentadas em três etapas: atividades de gerenciamento, atividades de assistência e humanização e atividades educativas:

1. Atividades de gerenciamento

- Estabelecer a estrutura organizacional do ambulatório: níveis hierárquicos (Figura 1) e organograma (Figura 2). - Organizar o fluxo de atendimento do cliente (Figura 3). - Adaptar o impresso de consulta médica, contendo: dados de identificação, anamnese, exame físico, campos para preenchimento de exames laboratoriais e de imagens e impresso de retorno.

- Criar folheto explicativo sobre a esquistossomose, utilizado durante aula educativa, ministrada a grupos de cinco clientes.

- Criar o almoxarifado do ambulatório, contendo materiais para serem utilizados no serviço, tais como, impressos, tubos de armazenamento de hemoderivados, drogas para tratamento da parasitose (oxaminiquine e praziquantel) e medicamentos para realização de exames diagnósticos (dulcolax ${ }^{\circledR}$ e fleet enema $\AA$ ).

- Implantar banco de dados dos clientes do ambulatório.

- Implantar cadastro eletrônico de coleta de materiais biológicos.

2. Atividades da assistência e humanização

- Implantar a consulta de enfermagem.

3. Atividades educativas
- Criar o Programa de Educação em Esquistossomose.

- Realizar aulas de reciclagem da equipe multidisciplinar.

\section{RESULTADO E DISCUSSÃO}

O Setor de Esquistossomose da Disciplina de Gastroenterologia atende, uma vez por semana, 30 clientes portadores de esquistossomose, nas suas diferentes formas anatomoclínicas. Em geral, são indivíduos na faixa etária de 20 a 40 anos, distribuição homogênea por parte da variável sexo, provenientes, em sua maior parte, da região Nordeste, com baixa renda familiar e pouca qualificação profissional ${ }^{(7)}$.

Com a introdução do enfermeiro no Setor, em 1998, foram levantados vários problemas de ordem administrativa e de assistência a essa população, que causavam atraso no atendimento e insatisfação do público. Os problemas de ordem administrativa identificados foram: o sistema de arquivo dos clientes constituía-se por impresso de difícil entendimento e com falta de espaço para seu preenchimento; o fluxo de atendimento não era controlado, não sendo respeitada a ordem de chegada dos clientes no ambulatório; a falta de informação aos clientes e a falta de clareza quando da marcação de exames, faziam com que retornassem desnecessariamente ao ambulatório.

Como medidas de ordem específica, foi necessária a criação de um serviço de enfermagem especializado que direcionasse a assistência ao cliente portador da esquistossomose. O pré-requisito para operacionalizar o serviço é a implementação da sistematização da assistência de enfermagem, tornandose fator fundamental para melhorar a qualidade do cuidado prestado ao cliente. A implantação dessa sistematização caracterizou-se pela criação do organograma e fluxograma do ambulatório e pela realização da consulta de enfermagem. Por meio dessa consulta, constatou-se déficit de conhecimento significativo sobre a esquistossomose por parte dos clientes. A partir desse referencial, a equipe começou a planejar o Programa de Educação à Esquistossomose ${ }^{(8)}$, direcionado ao cliente, familiares e amigos. Nesse aspecto, o serviço de enfermagem ficou constituído de atividades de gerenciamento, de assistência de humanização e de educação. 
Atividades de gerenciamento

O enfermeiro, atuando como gerente, desenvolve sua capacidade de organização, agregando toda equipe multidisciplinar e multiprofissional.

Organizar significa agrupar, estruturar e integrar os recursos organizacionais. Nesse âmbito, o enfermeiro tem papel fundamental na estrutura organizacional, pois, além de administrar, estabelece a divisão do trabalho da equipe, define os níveis de autoridade e de responsabilidade ${ }^{(2)}$. A Figura 1 ilustra o nível hierárquico do Setor, constituído por um chefe, médico e Professor adjunto, para a orientação dos demais profissionais de saúde (médicos, enfermeiros e psicóloga) no atendimento ao portador de esquistossomose. A seguir, encontramos os pós-graduandos de Medicina e de Enfermagem, responsáveis pela administração e organização do ambulatório, onde atuam residentes, estagiários, alunos de iniciação científica e monitores.

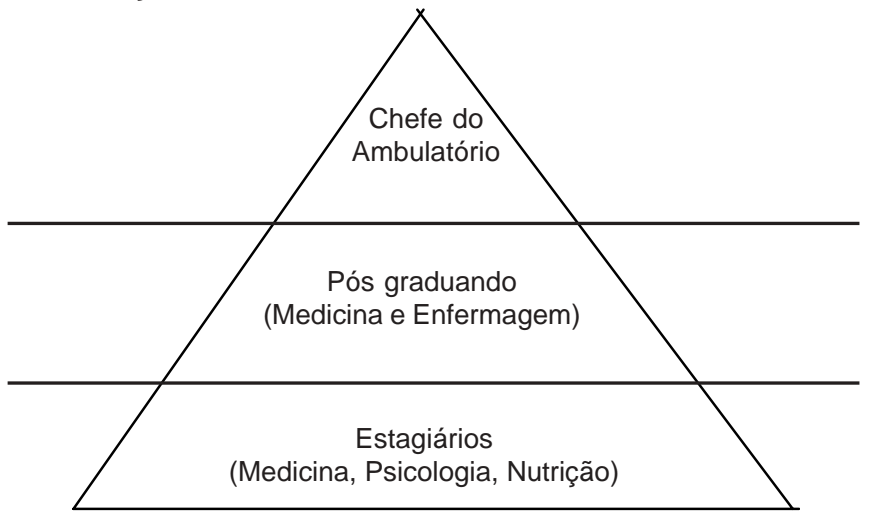

Monitores de Enfermagem (Alunos de Iniciação Científica)

Figura 1 - Níveis hierárquicos do ambulatório de esquistossomose

A divisão do trabalho é princípio fundamental para que a atividade profissional seja executada com mais eficiência $^{(2)}$. A Figura 2 ilustra esse fato por meio do organograma do Setor de Esquistossomose. A princípio, temos a figura do chefe do setor que atua nas decisões finais de toda a equipe multiprofissional e seleciona os componentes da equipe para os respectivos cargos e funções. $\mathrm{O}$ assistente I trabalha integradamente com o Supervisor de recursos humanos, enfermeiro, que supervisiona o serviço de enfermagem; promove reuniões periódicas com a equipe de enfermagem para planejar, organizar e verificar a qualidade de assistência de enfermagem prestada aos clientes.

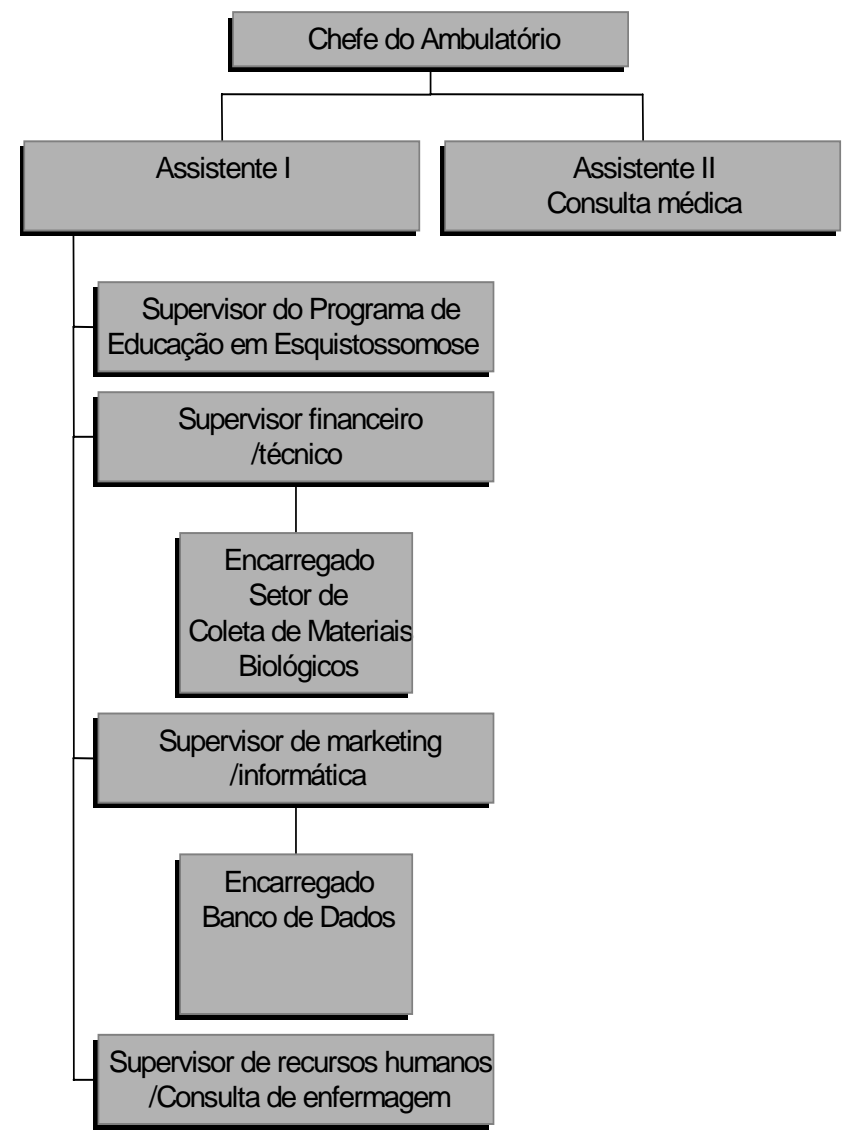

Figura 2 - Organograma do ambulatório de esquistossomose

Nesse mesmo nível hierárquico, temos o assistente II, médico, que avalia a assistência médica prestada aos clientes; colabora com a equipe multidisciplinar no estabelecimento de regime de trabalho eficaz e dinâmico. O supervisor do Programa de Educação em Esquistossomose, enfermeiro, aplica e avalia a qualidade da aula e dinâmica de grupo realizado durante o andamento do ambulatório. O supervisor financeiro/ técnico, enfermeiro, requisita, controla e registra os gastos materiais, equipamentos e produtos; mantém estoque de medicamentos (oxaminiquine, praziquantel, dulcolax ${ }^{\circledR}$, fleet enema ${ }^{\circledR}$ ) necessários para o bom funcionamento do setor. Ele é também responsável pelo encarregado da coleta de materiais biológicos, monitor de enfermagem, que providencia a coleta e o armazenamento adequado de materiais no congelador, com monitorização da temperatura. O supervisor de marketing/informática, enfermeiro, promove intercambio com o Posto de Saúde da Vila Mariana e o Hospital Pirajussara (conveniados com o Hospital São Paulo), para encaminhamento dos clientes portadores da esquistossomose e responsável pelo encarregado do banco de dados, monitor de enfermagem, que realiza o cadastro dos clientes no banco de dados, 
para automatizar algumas atividades burocráticas de escrituração, associadas à documentação administrativa e/ou assistencial.

Essa divisão de trabalho proporcionou a criação do almoxarifado, a implantação do projeto piloto do banco de dados do cliente e a criação de protocolos de atendimento e seguimento. Esses protocolos foram reformulados para oferecer atendimento que priorize as necessidades dos clientes e agilize o tempo de consulta.

A Figura 3 mostra o fluxograma geral. Os passos do cliente atendido no Setor iniciam-se no guichê para abertura da ficha de atendimento, sendo, em seguida, encaminhado à monitora de enfermagem responsável pela avaliação preliminar e análise do prontuário. Esses pacientes são convidados a participar do Programa de Educação em Esquistossomose e dinâmica de grupo, até o momento da consulta médica ou de enfermagem. Após a consulta, o profissional encaminha o cliente para a monitora de enfermagem responsável pela orientação pósconsulta. Essa atuação caracteriza-se pela explicação do preparo para exames, esclarecimento das rotinas burocráticas do hospital e coleta de material biológico, sendo, em seguida, liberado para dar continuidade ao seguimento ambulatorial.

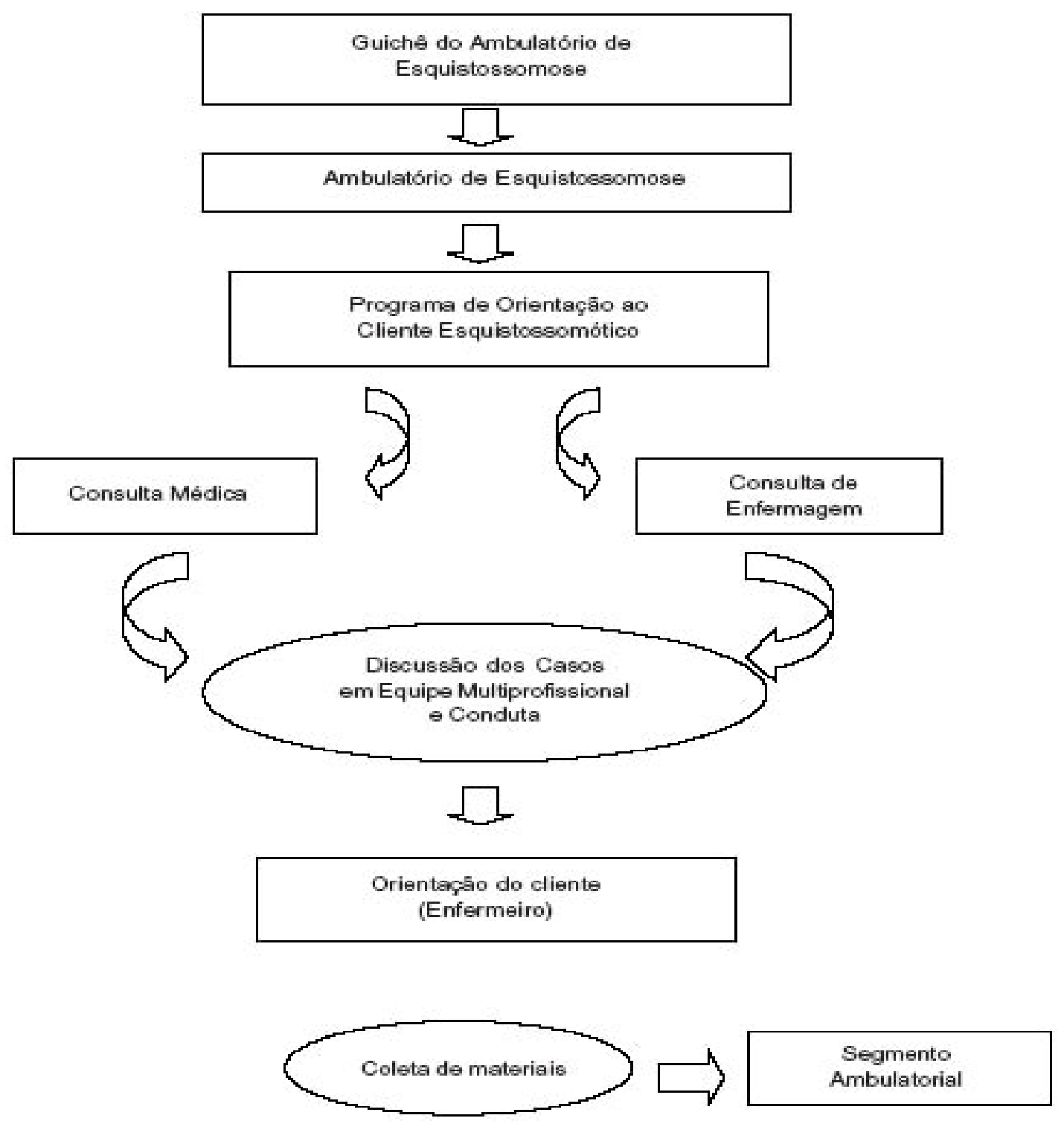

\section{Figura 3 - Fluxo do atendimento ao cliente}

Atividade de assistência e humanização

A estratégia utilizada foi adequar a necessidade da clientela ao processo de trabalho da enfermagem, por meio do atendimento clínico individual proporcionado pela consulta de enfermagem. Para isso, foi necessário elaborar protocolo de levantamento de dados, o qual permite identificar os diagnósticos de enfermagem para que seja possível desenvolver as intervenções necessárias.

A consulta de enfermagem tem seu enfoque na 
educação em saúde, no desenvolvimento de condição de bem estar pelo autocuidado e no entendimento do indivíduo como um todo (mente, corpo e espírito). Ela identifica situações de saúde/doença e prescreve e implementa medidas de enfermagem que contribuam para a promoção, prevenção e proteção da saúde, recuperação e reabilitação do indivíduo, da família e da comunidade ${ }^{(9-10)}$.

A implantação da consulta de enfermagem como parte da rotina do Setor nos trouxe, no ano de 2000, a realização de 64 consultas de enfermagem e de 169 consultas no ano de 2001.

Essa nova postura, respaldada por lei, incentiva a multidisciplinaridade com o objetivo de atender o indivíduo como um todo. Com isso, os enfermeiros vêm obtendo o respeito dos usuários e o reconhecimento por parte dos próprios profissionais da saúde, abrindo oportunidades para o crescimento da profissão.

No ano de 2002, foi realizado o primeiro trabalho sobre a assistência de enfermagem a portadores de esquistossomose nessa instituição. Foram identificandos 33 diagnósticos de enfermagem e 7 problemas colaborativos $^{(7)}$. Futuramente, será necessária a adequação e validação desses diagnósticos de enfermagem encontrados, analisando número maior de clientes. E, ainda, para cada diagnóstico, estaremos trabalhando nas orientações específicas (intervenção).

Consideramos que a experiência profissional adquirida com os pacientes portadores de esquistossomose permitirá a consolidação da assistência de enfermagem e complementará, cada vez mais, o atendimento ambulatorial.

Atividade educativa (Programa de Educação em Esquistossomose)

Por meio das consultas médicas e de enfermagem realizadas, constatou-se déficit de conhecimento significativo dos clientes sobre a esquistossomose. E, a

\section{REFERÊNCIAS BIBLIOGRÁFICAS}

1. Silva VEF. Administração em enfermagem. São Paulo (SP): Editora Universitária; 1991.

2. Chiavenatto I. Administração nos Novos Tempos. São Paulo (SP): MAKRON Books do Brasil; 1999.

3. Lunardi WDLF, Lunardi GL, Paulitsch FS. A prescrição de enfermagem computadorizada como instrumento de comunicação nas relações multiprofissionais e intra equipe de enfermagem: relato de experiência. Rev Latino-am Enfermagem 1997 julho; 5(3):63-9. partir desse referencial, a equipe planejou o Programa de Educação em Esquistossomose em que o público alvo fosse além dos clientes do ambulatório e de seus acompanhantes (familiares e amigos) ${ }^{(8)}$. Essa atividade vem sendo desenvolvida no Setor e está disponível na internet, no sítio www.unifesp.br/dmed/gastro/dgastro.htm.

A educação em saúde é considerada como sendo responsabilidade primária do enfermeiro e função independente na prática de enfermagem ${ }^{(11)}$. Essa atividade tem, entre seus objetivos, ensinar como se transmite e evolui a esquistossomose, esclarecer as principais dúvidas, favorecer a troca de experiência e enfatizar a importância do seguimento ambulatorial em seus diversos aspectos.

Independente da metodologia utilizada, é de fundamental importância que as práticas educativas tenham caráter participativo, permitindo a troca de informações e experiências baseadas na vivência do grupo. Nesse sentido, a utilização de linguagem acessível, simples e precisa pelo profissional de saúde deve ser sempre lembrada ${ }^{(12)}$.

\section{CONSIDERAÇÕES FINAIS}

As rotinas administrativas, implantadas em 1998, parecem ter contribuído, de forma significativa, para a organização do setor. As atividades administrativas, vale ressaltar, têm sido discutidas e avaliadas freqüentemente pela equipe, com o intuito de se detectarem possíveis falhas que implicarão novas mudanças. Essas mudanças têm como objetivo a organização do Setor e melhor atendimento do cliente. Buscamos, além do oferecimento de assistência qualificada, a compatibilização com seu custo, o que passou a ser um desafio para o setor. É imperativo o empenho de todos em fazer uso adequado de recursos econômicos e financeiros, de pessoal e materiais disponíveis, voltados para oferecer o melhor atendimento aos clientes, a custos suportáveis.

4. Silva EM, Nozawa MR, Silva JC, Carmona SAMLD. Práticas das enfermeiras e políticas de saúde pública em Campinas, São Paulo, Brasil. Cad Saúde Publica 2001; 17(4):989-98. 5. Dias MAA, Juice K. Primary Nursing - Qualidade no cuidado de enfermagem. In: Michel JLM, Yassuda, D, Hirose, HMU, Matsushita, MS, coordenadores. Investindo no desenvolvimento de enfermeiros de hospitais de ensino. $\mathrm{V}$ Encontro de enfermeiros de hospitais de ensino do Estado de São Paulo; 1999. Março 11-13; São Paulo, São Paulo. São Paulo; 1999. p. 249.

6. Coren - Conselho Regional de Enfermagem. Resolução COFEN - 159: Dispõe sobre a Consulta de Enfermagem. São Paulo; 2001. 
7. Kubo $\mathrm{CH}$. Assistência de enfermagem a portadores de esquistossomose: levantamento de dados e diagnósticos de enfermagem em um serviço de saúde especializado. [tese]. São Paulo: Universidade Federal de São Paulo; 2002. 8. Ribeiro PJ. Desenvolvimento e avaliação de um Programa Educativo para Portadores de Esquistossomose. [tese]. São Paulo: Universidade Federal de São Paulo; 2002.

9. Lacava RM do VB. Elaboração de um protocolo de levantamento de dados e diagnósticos de enfermagem em gestantes. [tese]. São Paulo: Universidade Federal de São Paulo; 1999.

10. Gerk MAS. Saúde da mulher: conjunto prioritário de diagnósticos de enfermagem em ginecologia [tese]. São Paulo: Universidade Federal de São Paulo; 2000.

11. Smeltzer SC, Bare BG. Tratado de enfermagem médicocirúrgica. Rio de Janeiro (RJ): Editora Guanabara Koogan; 1994.

12. Ministério da Saúde (BR). Assistência ao planejamento familiar. Brasília (DF): Secretaria Nacional de Programas Especiais, Ministério da Saúde; 1987. 